\title{
Risks of iron deficiency among vegetarian college women
}

\author{
Susan N. Hawk ${ }^{1^{*}}$, Kimberly Grage Englehardt ${ }^{2}$, Cindi Small ${ }^{2}$ \\ ${ }^{1}$ Department of Nutrition, Health and Exercise Science, Central Washington University, Ellensburg, USA; \\ *Corresponding Author: woodys@cwu.edu \\ ${ }^{2}$ Department of Food Science and Nutrition, California Polytechnic State University, San Luis Obispo, USA
}

Received 2 December 2011; revised 30 December 2011; accepted 9 January 2012

\begin{abstract}
Iron deficiency anemia is the most common nutritional deficiency disease worldwide and poses a major threat in women of child-bearing age and those who follow a vegetarian diet. The objective of this study was to ascertain whether differences exist in iron status markers between female university students following a vegetarian and nonvegetarian diet. This study took a cross sectional analysis of $\mathbf{3 9}$ female students at California Polytechnic State University (Cal Poly State University) in San Luis Obispo, CA between the ages of 18 and 22. Of the participants, 19 followed a vegetarian diet and 20 followed a nonvegetarian diet. Characteristic, demographic, and anthropometric data were collected and analyzed. The results showed no significant difference in iron intake between the two groups. However, nearly $66 \%$ of vegetarians and $65 \%$ of non-vegetarians failed to meet the Recommended Daily Allowance for iron. No significant difference was found for serum iron, serum ferritin, transferrin saturation, and total iron binding capacity between subjects. Serum ferritin tended to be lower for vegetarians compared to non-vegetarians. Both vegetarians and non-vegetarians were in stage IV negative iron balance, with more vegetarians tending to be in stage IV negative iron balance than non-vegetarians. Thus, female college students, irrespective of their meat intake, may be at higher risk of developing negative iron balance and should be educated about iron deficiency anemia and the prevention of iron depletion.
\end{abstract}

Keywords: Iron; Anemia; Vegetarian; Negative Iron Balance

\section{INTRODUCTION}

Iron deficiency anemia is the most prevalent nutritional deficiency disease worldwide and is often most commonly seen in children and women of child-bearing age [1]. In fact, estimates assume that impaired iron status occurs in $1 \%$ to $6 \%$ of the general population [2]. Regardless of dietary practices, the iron status of women is inferior to men [3]. Roughly $5 \%$ to $14 \%$ of women in the United States ages 15 to 44 years have impaired iron status [2]. Young women may be at the greatest risk of developing early stages of iron depletion as evidenced by one study that noted the prevalence to be $16 \%$ in women ages 20 to 49 years compared to $5 \%$ in women ages 50 to 79 [4]. Even marginal iron status is a potential problem in women and may pose a particular risk to those consuming either a vegan or vegetarian diet [5].

The dietary factors of greatest influence over one's iron status include the form of iron consumed and any factors affecting its bioavailability [4]. In support of this, the 2010 Dietary Guidelines for Americans recommends that women of childbearing age eat foods rich in hemeiron and consume vitamin $\mathrm{C}$-rich foods to enhance iron uptake [6]. Given that women of child bearing age tend to consume a diet limited in heme-iron, vegetarian college-aged women may be at considerable risk for poor iron status and anemia. The objective of this study was to ascertain whether college-aged vegetarian women have compromised iron status as indicated by altered hemoglobin, serum iron, serum ferritin, transferrin saturation, and total iron binding capacity.

\section{METHODS}

\subsection{Subjects}

Subjects were recruited from a convenience sample of students at California Polytechnic State University (Cal Poly), San Luis Obispo. Participants were female, current Cal Poly students between the ages of 18 and 22 . Excluded from the study were women who smoked, were pregnant, took vitamin or mineral supplements, or took any medications (including oral contraceptives). Each individual interested in participating was interviewed and 
asked to complete a questionnaire to determine if they met all of the inclusion criteria. Thirty-nine subjects met the criteria and participated in this study. Among the subjects, 19 were self-reported vegetarians and 20 were self-reported non-vegetarians. Vegetarians were defined as those who did not consume meat or fish for approximately one year prior to and during the study. Of the vegetarians, three followed a vegan diet and one followed an ovo-vegetarian diet.

The research project was pre-approved by the Food Science and Nutrition Department, Research and Graduate Programs, and the Cal Poly Human Subjects Committee. All participants read and signed an informed consent for the participation in this research study.

\subsection{Questionnaire}

Eligible participants completed a questionnaire and a follow-up interview was conducted based on the responses given. Information including age, major, grade level, menstrual cycle, eating out habits, physical activity, diet, and any previous diagnosis of anemia was gathered frequency questionnaire regarding their consumption rates of proteins, starches, fruits, vegetables, dairy products, fats, oil, and sweets. Information gathered from the food frequency questionnaire was used to verify and validate classification of each participant's self-reported diet type.

\subsection{Anthropometric Measures}

Anthropometric measurements were taken at the initial participant meeting. Weight and height were measured and used to calculate body mass index (BMI).

\subsection{Dietary Intake}

The initial participant meeting also included instructtions for a three day food record. The participants were instructed to record all food and fluid consumed over two typical nonconsecutive weekdays and one typical weekend day. Other instructions provided addressed recording time, type and amount of food consumed, use of separate food record sheets for each day, recording brand names of food, ingredients, and names of restaurants. Food models were used to demonstrate portion sizes, household measures were discussed, food labels were reviewed and food reference materials were given to each participant to provide a better understanding on how to estimate portions of food and fluid consumed. Food records were reviewed by the researchers together with the participant to ensure that the information had been properly described. The three day food records were analyzed for each participant via Food Processor SQL Edition Version 9.6.0. The program included food items traditionally consumed in the United States. Mixed dishes were often separated into basic food components and participants provided the researchers with food labels or detailed descriptions when uncommon foods were consumed. The data was then used to ascertain differences in nutrient intakes between vegetarians and non-vegetarians. The data analyzed included the consumption of calories, fat, cholesterol, carbohydrates, sugar, fiber, protein, iron, vitamin $\mathrm{C}$, calcium, phosphorus, caffeine, and water.

\subsection{Hematological Analysis}

A 12 hour fasting blood sample was drawn the week prior to each participant's menstrual cycle and analyzed for hematological indices. Measures included white blood cells, red blood cells, hemoglobin, hematocrit, mean corpuscular volume, mean corpuscular hemoglobin concentration, red cell diameter width, total protein, albumin, triglyceride, cholesterol, HDL, LDL, total cholesterol/HDL ratio, serum iron, serum ferritin, and total iron binding capacity (TIBC). Transferrin saturation was also assessed.

\subsection{Statistical Analysis}

The statistical analysis was carried out using SAS for Windows Version 9.1. Results were considered significant at $p \leq 0.05$. Results were given as means with standard deviations (SD), unless otherwise noted. Nonparametric techniques were used to avoid violations with normal distribution assumptions, due to varying distributions of the parameters and small sample size. For demographics and baseline characteristics, the MannWhitney Wilcoxon statistical test was used to assess any differences among subjects. For the analysis of selected nutrient and hematological parameters, the Spearman Rank Correlation statistical test for associations was used. Fisher's Exact statistical test was used for associations between vegetarians and non-vegetarians. This included associations with the percent of vegetarians versus nonvegetarians meeting the Recommended Dietary Allowance and Estimated Average Requirement for iron intake, and various hematological cut-off values determining iron status.

\section{RESULTS}

\subsection{Subject Characteristics and Demographics}

Subject characteristic and demographic information from the initial questionnaire are summarized in Table 1. Undergraduate participants represented 17 different majors. Four non-vegetarians and three vegetarians either had a prior history of anemia or suspected that they may be anemic. The number of individuals who consumed meals from restaurants was similar between the two groups, with only one non-vegetarian eating in exclusively. The non-vegetarian participants were older in age at a mean 
Table 1. Demographics of participants.

\begin{tabular}{cccccc}
\hline & \multicolumn{2}{c}{$\begin{array}{c}\text { Nonvegetarians } \\
(\mathrm{n}=20)\end{array}$} & \multicolumn{2}{c}{$\begin{array}{c}\text { Vegetarians } \\
(\mathrm{n}=19)\end{array}$} \\
\cline { 2 - 6 } & Mean & SD $(+/-)$ & Mean & SD $(+/-)$ & p-value \\
\hline Age (years) & 20.65 & 1.04 & 19.68 & 1.57 & 0.05 \\
Height (inches) & 65.32 & 3.42 & 65.30 & 2.63 & 0.68 \\
Weight (pounds) & 144.30 & 32.77 & 135.40 & 23.52 & 0.38 \\
BMI $\left(\mathrm{kg} / \mathrm{m}^{2}\right)$ & 23.75 & 4.55 & 22.53 & 4.06 & 0.45 \\
\hline
\end{tabular}

Significance was noted at $\mathrm{p} \leq 0.05$.

of 20.7 years compared to the vegetarians at a mean age of 19.7 years $(p=0.05)$. Height for the two groups was similar, with a mean height of 65.3 inches for both groups $(p=0.68)$. Weight and BMI for the vegetarians tended to be lower, albeit not significantly, compared to non-vegetarians. The BMIs were within the normal limits for both groups. Exercise patterns were similar among subjects.

\subsection{Dietary Intake}

Vegetarians tended to consume more dietary fiber, soluble fiber, and monounsaturated fat compared to nonvegetarians (Table 2). Non-vegetarians did consume more daily protein than vegetarians, $76.9 \mathrm{~g}$ per vs. $58.7 \mathrm{~g}$, respectively $(\mathrm{p}=0.01)$. They also tended to consume greater calories than vegetarians as evidenced by examining weight of food consumed, total calories, calories from fat, calories from saturated fat, fat intake, polyunsaturated fat, saturated fat, trans fatty acids, cholesterol, carbohydrates, total sugars, caffeine, and water consumption compared to vegetarians. There was no significant difference in iron consumption between groups. However, non-vegetarians tended to have higher iron intake than vegetarians, $16.8 \mathrm{mg}$ compared to $14.8 \mathrm{mg}$, respectively. Nutrients known to influence iron bioavailability including vitamin $\mathrm{C}$, calcium, and phosphorus tended to be higher in the non-vegetarian participants.

\subsection{Daily Reference Intake of Iron}

The number of vegetarians and non-vegetarians meeting the Recommended Dietary Allowance (RDA) and Estimated Average Requirement (EAR) were compared (Table 3). Based on the Dietary Reference Intakes (DRI) for iron in females aged 14 to 18 , seven vegetarians $(85.7 \%)$ were under the RDA and two (28.6\%) fell below the EAR for iron intake. Based on the DRI for iron in females aged 19 to 30 years, $66.7 \%$ of vegetarians compared to $65 \%$ of non-vegetarians failed to meet the RDA. There were slightly more non-vegetarians at $10 \%$ compared to vegetarians at $8.3 \%$ not meeting the EAR for iron intake. Overall, fewer participants consumed below the Estimated Average Requirement compared to the Recommended Dietary Allowance.
Table 2. Selected daily nutrient intakes vegetarians and nonvegetarians.

\begin{tabular}{lccccc}
\hline \multirow{2}{*}{\multicolumn{1}{c}{ Nutrient Intake }} & \multicolumn{2}{c}{$\begin{array}{c}\text { Non-vegetarian } \\
(\mathrm{n}=20)\end{array}$} & \multicolumn{2}{c}{$\begin{array}{c}\text { Vegetarian } \\
(\mathrm{n}=19)\end{array}$} \\
\cline { 2 - 6 } & Mean & $\mathrm{SD}(+/-)$ & Mean & $\mathrm{SD}(+/-)$ & $\mathrm{p}$-value \\
\hline Density (g) & 2566.80 & 882.90 & 2290.90 & 871.40 & 0.38 \\
Calories (kcal) & 1917.50 & 513.60 & 1655.80 & 626.90 & 0.13 \\
Calories from Fat (kcal) & 555.80 & 215.50 & 511.70 & 252.70 & 0.58 \\
Calories from Sat Fat & 182.40 & 79.87 & 146.70 & 88.41 & 0.19 \\
(kcal) & 61.76 & 23.94 & 56.96 & 28.18 & 0.60 \\
Fat (g) & 13.14 & 7.10 & 14.95 & 10.19 & 0.76 \\
Monounsaturated fat (g) & 6.72 & 4.62 & 7.92 & 4.29 & 0.27 \\
Polyunsaturated Fat (g) & 20.27 & 8.87 & 16.30 & 9.82 & 0.20 \\
Saturated Fat (g) & 0.22 & 0.37 & 0.66 & 0.74 & 0.41 \\
Trans fatty acids (g) & 176.10 & 89.79 & 154.40 & 178.50 & 0.11 \\
Cholesterol (mg) & 268.80 & 83.51 & 238.80 & 91.45 & 0.32 \\
Carbohydrates (g) & 94.92 & 37.61 & 80.36 & 42.96 & 0.22 \\
Total Sugars (g) & 21.62 & 10.43 & 25.98 & 13.35 & 0.31 \\
Dietary Fiber (g) & 1.69 & 1.37 & 2.45 & 1.53 & 0.12 \\
Soluble Fiber (g) & 76.99 & 24.93 & 58.73 & 29.10 & 0.01 \\
Protein (g) & 16.82 & 6.36 & 14.84 & 7.10 & 0.48 \\
Iron (mg) & 129.90 & 58.52 & 128.60 & 153.20 & 0.10 \\
Vitamin C (mg) & 961.50 & 330.50 & 903.40 & 447.60 & 0.35 \\
Calcium (mg) & 763.00 & 246.10 & 662.80 & 293.70 & 0.27 \\
Phosphorus (mg) & 89.24 & 72.70 & 58.69 & 43.54 & 0.39 \\
Caffeine (mg) & 1853.70 & 764.00 & 1629.70 & 797.00 & 0.35 \\
Water (g) & & & & & \\
\hline & & & &
\end{tabular}

Significant was noted at $\mathrm{p} \leq 0.05$.

Table 3. Iron intake compared to RDA.

\begin{tabular}{llll}
\hline & & Non-vegetarians & Vegetarians \\
\hline \multirow{2}{*}{ RDA } & $14-18$ yrs $(\mathrm{n}=7) \quad(\mathrm{n}=0)$ & $85.7 \%(\mathrm{n}=6)$ \\
& $19-30$ yrs $(\mathrm{n}=32) 65 \%(\mathrm{n}=13)$ & $66.7 \%(\mathrm{n}=8)$ \\
$<$ EAR & $14-18$ yrs $(\mathrm{n}=7) \quad(\mathrm{n}=0)$ & $28.6 \%(\mathrm{n}=2)$ \\
& $19-30$ yrs $(\mathrm{n}=32) 10 \%(\mathrm{n}=2)$ & $8.3 \%(\mathrm{n}=1)$ \\
\hline
\end{tabular}

Note: Percentages for non-vegetarian females aged 14 to 18 years were not determined because there were no participants in this age bracket.

\subsection{Hematological Analysis}

Baseline hematological parameters were compared among participants (Table 4). Vegetarians had significantly higher albumin $(p=0.02)$, mean corpuscular hemoglobin concentration $(\mathrm{MCHC})(\mathrm{p}=0.04)$, and high density lipoprotein cholesterol (HDL) $(p=0.02)$ compared to non-vegetarians. The vegetarians also tended to have higher red cell distribution width (RDW) compared to the non-vegetarians, albeit statistically insignificant. The non-vegetarians had significantly higher hematocrit $(\mathrm{p}=$ $0.03)$, total protein $(\mathrm{p}=0.02)$, low density lipoproteins cholesterol $(\mathrm{LDL})(\mathrm{p}=0.03)$, and total cholesterol/HDL ratio $(p=0.002)$. Non-vegetarians tended to have higher white and red blood cell counts, hemoglobin, mean corpuscular volume (MCV), triglycerides, and cholesterol compared to vegetarians, although these differences were 
Table 4. Hematological values for vegetarians and non-vegetarians.

\begin{tabular}{lccc}
\hline & Non-vegetarian & Vegetarian & p-value \\
\hline $\begin{array}{l}\text { White Blood Cells } \\
\text { (thousand cells/mcL) }\end{array}$ & $6.19 \pm 1.21$ & $5.37 \pm 1.21$ & 0.08 \\
Red Blood Cells & & & \\
(million cells/mcL) & $4.42 \pm 0.23$ & $4.38 \pm 0.41$ & 0.90 \\
HgB (g/dL) & $13.39 \pm 0.76$ & $12.94 \pm 1.19$ & 0.19 \\
Hct $(\%)$ & $40.09 \pm 2.09$ & $38.16 \pm 3.17$ & 0.03 \\
MCV (fl) & $90.81 \pm 2.99$ & $87.58 \pm 6.53$ & 0.08 \\
MCHC (g/dL) & $33.39 \pm 0.50$ & $33.90 \pm 1.15$ & 0.04 \\
RDW (\%) & $12.23 \pm 0.55$ & $12.77 \pm 1.96$ & 0.55 \\
Total Protein (g/dL) & $7.39 \pm 0.30$ & $7.12 \pm 0.36$ & 0.02 \\
Albumin $(g / d L)$ & $4.14 \pm 0.27$ & $4.35 \pm 0.23$ & 0.02 \\
Triglyceride (mg/dL) & $72.50 \pm 32.90$ & $70.00 \pm 29.18$ & 0.94 \\
Cholesterol (mg/dL) & $153.20 \pm+20.58$ & $150.50 \pm 20.43$ & 0.72 \\
HDL (mg/dL) & $54.30 \pm 14.30$ & $65.05 \pm 11.06$ & 0.02 \\
LDL (mg/dL) & $84.35 \pm+14.44$ & $71.42 \pm 18.30$ & 0.03 \\
Total Cholesterol/HDL & $2.94 \pm 0.52$ & $2.37 \pm 0.48$ & 0.00 \\
Serum Iron ( $\mu \mathrm{g} / \mathrm{dL})$ & $102.30 \pm 46.72$ & $81.47 \pm 35.58$ & 0.12 \\
Serum Ferritin $(\mathrm{ng} / \mathrm{mL})$ & $27.75 \pm 18.01$ & $23.16 \pm 15.54$ & 0.47 \\
Transferrin Saturation $(\%)$ & $28.85 \pm 15.17$ & $23.10 \pm 11.31$ & 0.24 \\
TIBC $(\mu \mathrm{g} / \mathrm{dL})$ & $374.60 \pm 62.29$ & $366.50 \pm 47.81$ & 0.57 \\
\hline
\end{tabular}

Significant was noted at $\mathrm{p} \leq 0.05$.

not statistically significant. Iron status indicators revealed serum iron, serum ferritin, transferrin saturation, and total iron binding capacity to tend to be greater for non-vegetarians, however there was no significant difference between the two groups.

\subsection{Serum Ferritin}

Only $10.5 \%$ of vegetarians, compared to $25 \%$ of nonvegetarians were within the normal range of serum ferritin (Figure 1). When looking at cut off values for serum ferritin, a high percentage of vegetarians were in negative iron balance. A greater number of vegetarians had ferritin values of $<25 \mathrm{ng} / \mathrm{mL}$, indicating stage I or early negative iron balance, $52.6 \%$ compared to non-vegetarians at $45 \%$. Serum ferritin values of $<20 \mathrm{ng} / \mathrm{mL}$, indicating stage II or iron depletion also tended to be higher for vegetarians at $47.4 \%$ compared to non-vegetarians at $40 \%$. Interestingly, $21.1 \%$ of vegetarians and $15 \%$ of non-vegetarians had serum ferritin levels $<10$ $\mathrm{ng} / \mathrm{mL}$, indicating stage III iron deficiency or stage IV iron deficiency.

\subsection{Iron Balance}

Serum ferritin, serum iron, total iron binding capacity (TIBC), and transferrin saturation levels for stage I, II, III, and IV negative iron balance are provided in Table 5. With respect to negative I iron balance, there was a significantly higher percentage of vegetarians $(94.7 \%)$ with serum iron of $<120 \mu \mathrm{g} / \mathrm{dL}$ compared to non-vegetarians

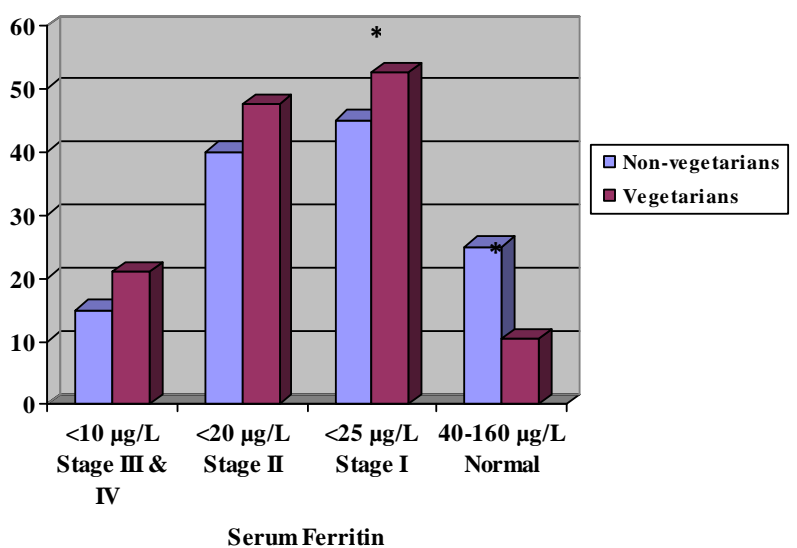

Note: An asterisk represents statistical significance between vegetarians and non-vegetarians for a single classification of serum iron levels.

Figure 1. Percentage of nonvegetarians and vegetarians with either normal serum ferritin levels or in stage I, II, III/IV of negative iron balance.

Table 5. Percentage of vegetarians and non-vegetarians with markers of poor iron status.

\begin{tabular}{ccccc}
\hline \multicolumn{2}{c}{ Stage Variable } & Non-vegetarians & Vegetarians & p-value \\
\hline \multirow{3}{*}{ Negative I } & Iron & $65 \%$ & $94.7 \%$ & 0.03 \\
& TIBC & $60 \%$ & $52.6 \%$ & 0.87 \\
& Saturation & $50 \%$ & $68.4 \%$ & 0.11 \\
& Ferritin & $45 \%$ & $52.6 \%$ & 0.44 \\
& Iron & $65 \%$ & $84.2 \%$ & 0.16 \\
& TIBC & $60 \%$ & $52.6 \%$ & 0.87 \\
& Saturation & $50 \%$ & $68.4 \%$ & 0.11 \\
& Ferritin & $40 \%$ & $47.4 \%$ & 0.44 \\
& Iron & $25 \%$ & $26.3 \%$ & 0.61 \\
& TIBC & $35 \%$ & $21.1 \%$ & 0.95 \\
& Saturation & $20 \%$ & $21.1 \%$ & 0.62 \\
& Ferritin & $15 \%$ & $21.1 \%$ & 0.47 \\
& Iron & $10 \%$ & $21.1 \%$ & 0.31 \\
& TIBC & $15 \%$ & $21.1 \%$ & 0.47 \\
& Saturation & $20 \%$ & $21.1 \%$ & 0.62 \\
& Ferritin & $15 \%$ & $21.1 \%$ & 0.47 \\
\hline
\end{tabular}

Significance was noted at $\mathrm{p} \leq 0.05$.

$(65 \%)(p=0.03)$. More vegetarians tended to have a transferrin saturation $>60 \%$ and serum ferritin $<25$ $\mathrm{ng} / \mathrm{mL}$ compared to non-vegetarians. In classifying negative II iron balance, there was a higher percentage of vegetarians with serum iron $<115 \mu \mathrm{g} / \mathrm{dL}$ and serum ferritin $<20$; commensurate with a lower percentage of vegetarians at or above the normal total iron binding capacity cut-off of $>360 \mu \mathrm{g} / \mathrm{dL}$ compared to non-vegetarians. For negative III iron balance, there were a higher percentage of vegetarians with serum iron $<60 \mu \mathrm{g} / \mathrm{dL}$, transferrin saturation $<15 \%$, serum ferritin $<10 \mathrm{ng} / \mathrm{mL}$, and a lower percentage of vegetarians with total iron binding capacity of $>390 \mu \mathrm{g} / \mathrm{dL}$ compared to non-vegetarians. Both vegetarians and non-vegetarians displayed 
signs of being in stage IV. Among vegetarians $21.1 \%$ had a serum iron value $<40 \mu \mathrm{g} / \mathrm{dL}$ and total iron binding capacity of $>410 \mu \mathrm{g} / \mathrm{dL}$ while only $10 \%$ of non-vegetarians had a serum iron $<40 \mu \mathrm{g} / \mathrm{dL}$ and $15 \%$ had a total iron binding capacity of $>410 \mu \mathrm{g} / \mathrm{dL}$. Comparisons of the percentage of vegetarians and non-vegetarians in negative II, III, and IV iron balance were not significantly different.

\section{DISCUSSION}

Given that young people often adopt a vegetarian lifestyle out of health, ethical, religious or environmental concerns, it is not surprising that such individuals show a stronger dietary profile than non-vegetarians. College women who ate an exclusively plant based diet consumed more monounsaturated fat, fewer calories from fat, less cholesterol, less sugar and less caffeine than nonvegetarians. These findings are consistent with other studies of dietary patterns of male and female vegetarians [7]. Of interest in our study was the elevated consumption of total dietary fiber and soluble fiber among vegetarians. This poses a concern due the inhibiting effects fiber has on iron absorption [8,9].

Although not statistically significant, vegetarians tended to consume less iron than omnivores. Our findings are consistent with most others noting no significant difference in iron intake among vegetarians and omnivores [10-12]. Yet, other findings have revealed a greater iron intake among vegetarians [7]. Part of this discrepancy may be attributable to iron food fortification. Worth noting is that since vegetarians often consume non-heme iron sources, they are susceptible to encountering bioavailability problems. Thus, iron intake is unlikely be directly correlated with iron status. In the case of this study, an assumption can be made that iron status may actually be poorer than the dietary intake would suggest.

In a study conducted in Taiwan, a typically non-iron fortifying country, Huang et al. [13] found non-vegetarians to have a significantly higher intake of iron and corresponding serum ferritin levels compared to vegetarians. The participants also used supplements which were recorded into the dietary intake and may have contributed to the higher ferritin levels. These results are contrary to those of Hadded et al. who investigated the iron status of vegans in Loma Linda, California [14]. In this case, the mean iron intake and serum ferritin levels were the same among non-vegetarian and vegan women [14]. Supplement use by participants was recorded and possibly aided in the higher iron intake and serum ferritin levels of the vegan participants [14].

When analyzing their dietary patterns, the participants on the vegetarian diet also consumed less protein and tended to have a lower intake of calcium, phosphorus, vitamin $\mathrm{C}$, and calories than non-vegetarians. While the amount of protein consumed daily was significantly lower in vegetarians $(58.7 \mathrm{~g})$ compared to non-vegetarians $(76.9 \mathrm{~g})$, this was still well above the Recommended Dietary Allowance (RDA) of 46 grams per day [6]. Calcium and phosphorus are both known inhibitors of iron absorption [12] but were not affecting iron status in our subjects given the low intake of both minerals among vegetarians $(903.4 \mathrm{mg}$ and $662.8 \mathrm{mg}$, respectively). These levels fell short of the RDAs of $1,000 \mathrm{mg}$ calcium and $700 \mathrm{mg}$ phosphorous per day, for females aged 19 to 50 years [16]. The discrepancy in calorie intake among subject groups may be reflective of women opting to be vegetarian as a means to weight loss. With respect to iron status and dietary intake, our findings show a positive association between iron intake, vitamin $\mathrm{C}$ intake and serum iron levels. Consistent with the literature and evidence of the enhancing effects of vitamin $\mathrm{C}$ on iron bioavailability, both iron and vitamin $\mathrm{C}$ intakes were negatively associated with total iron binding capacity [10].

Upon evaluation of hematological parameters, the data showed that vegetarians had stronger lipid profiles than non-vegetarians as evidenced by lower triglycerides, cholesterol, LDL, total cholesterol/HDL ratio, and higher HDL. These values which are closely tied to reduced risk of cardiovascular disease, are in accordance with other findings of lower mortality for ischemic heart disease in this population [17]. With respect to RDW, taking into account the standard deviation, some individual vegetarians could have had a RDW of approximately 14.7; a value greater than the often used upper/abnormal cut-off of $14 \%$. Thus, this would seem to indicate that at least a few of the vegetarians have an abnormal red blood cell size distribution, raising the question of whether this is reflective of the first signs of microcytic cells. Since serum ferritin is an optimal marker of iron status [9,18-20], we were intrigued by the data showing only $10.5 \%$ of the vegetarians versus $25 \%$ of non-vegetarians to be within normal limits for serum ferritin. Similar to other research findings, there were no significant differences between serum ferritin and iron intake [21-22]. In fact, there was a positive correlation between iron intake and serum ferritin levels. As serum ferritin decreased total iron binding capacity increased. Vegetarians also tended to have lower serum iron, total iron binding capacity and transferrin saturation, albeit not statistically significant. Of greatest interest was the finding that $21 \%$ of vegetarians and $15 \%$ of non-vegetarians were in stage IV negative iron balance. This difference was not statistically significant, yet is noteworthy given that stage IV directly precedes iron deficiency anemia. It appears that college age women are at risk for stage IV negative iron balance and potentially iron deficiency anemia, irrespective of whether or not they are vegetarian. 
Typically, hemoglobin status is positively correlated with iron intake [23]. This was not evident in our study and may be due to our sample size. However, studies of vegetarians typically show no indication of suboptimal hemoglobin, hematocrit, serum iron, total iron binding capacity or transferrin saturation when compared to nonvegetarians [10-12]. The lack of correlation between dietary iron intake and serum iron may also be attributable to diurnal fluctuations [19].

Menstrual blood loss can also be a contributing factor to the low iron status in women $[24,25]$ We attempted to eliminate this as a casual factor by scheduling participant blood draws approximately one week prior to the subject's menses cycle. However, irregular menstrual cycles were a concern for $25 \%$ of non-vegetarians and $26 \%$ of vegetarians, with one being amenorrheic. Based on hematological indicators of iron status we can conclude that female college students may be at increased risk for developing iron deficiency anemia.

\section{ACKNOWLEDGEMENTS}

This research was sponsored by Faculty Development and Faculty Support grants from California Polytechnic State University, San Luis Obispo, CA.

\section{REFERENCES}

[1] World Health Organization (2008) Worldwide prevalence of anaemia 1993-2005. http://whqlibdoc.who.int/publications/2008/97892415966 57 end.pdf

[2] Messina, M. and Messina, V. (1996) The Dietitian's guide to vegetarian diets issues and applications. Ballantine Books a division of Randon House Inc., New York.

[3] Shaw, N., Chin, C. and Pan, W. (1995) A vegetarian diet rich in soybean products compromises iron status in young students. Journal of Nutrition, 125, 212-219.

[4] Brussaard, J.H., Brants, H.A.M., Bouman, M. and Lowik, M.R.H. (1997) Iron intake and iron status among adults in the Netherlands. European Journal of Clinical Nutrition, 51, 51-58.

[5] Haddad, E.H., Berk, L.S., Kettering, J.D., Hubbard, R.W. and Peters, W.R. (1999) Dietary intake and biochemical, hematologic, and immune status of vegans compared with nonvegetarians. The American Journal of Clinical Nutrition, 70, 586-593.

[6] United States Department of Health and Human Services (U.S.DHHS) (2010) Dietary guidelines for Americans. http:/www.cnpp.usda.gov/DGAs2010-PolicyDocument.ht $\underline{\mathrm{m}}$

[7] Craig, J.W. (2011) Nutrition concerns and health effects of vegetarian diets. Nutrition in Clinical Practice, 25, 613-620. doi:10.1177/0884533610385707

[8] Harman, S.K. and Parnell, W.R. (1998) The nutritional health of New Zealand vegetarians and non-vegetarians seventh-day adventists: Selected vitamins, minerals, and lipid levels. The New Zealand Medical Journal, 111, 9194.

[9] Pongstaporn, W. and Bunyaratavej, A. (1999) Hematological parameters, ferritin, and vitamin B12 in vegetarians. Journal of Medical Association of Thailand, 82, 304-311.

[10] Donovan, U.M. and Gibson, R.S. (1995) Iron and zinc status of young women aged 14 to 19 years consuming vegetarian and omnivorous diets. Journal of the American College of Nutrition, 14, 463-472.

[11] Helma, Ad. and Darnton-Hill, I. (1987) Vitamin and iron status in new vegetarians. American Journal of Clinical Nutrition, 45, 785-789.

[12] Worthington-Roberts, B.S., Breskin, M.W. and Monsen, E.R. (1988) Iron status of premenopausal women in a university community and its relationship to habitual dietary sources of protein. American Journal of Clinical Nutrition, 47, 275-279.

[13] Huang, Y.C., Lin, W.J., Cheng, C.H. and Su, K.H. (1999) Nutrient intakes and iron status of healthy young vegetarians and non-vegetarians. Nutritional Research, 19, 663-674. doi:10.1016/S0271-5317(99)00031-7

[14] Haddad, E.H., Berk, L.S., Kettering, J.D., Hubbard, R.W. and Peters, W.R. (1999) Dietary intake and biochemical, hematologic, and immune status of vegans compared with non-vegetarians. The American Journal of Clinical Nutrition, 70, 586-593.

[15] Hallberg, L. and Hulthen, L. (2000) Prediction of dietary iron absorption: An algorithm for calculating absorption and bioavailability of dietary iron. The American Journal of Clinical Nutrition, 71, 1147-1160.

[16] Food and Nutrition Board Institute of Medicine (2010) Dietary reference intakes for calcium, phosphorous, magnesium, vitamin D and fluoride. National Academy Press, Washington DC.

[17] Key, T.J., Fraser, G.E., Thorogood, M., Appleby, P.N., Beral, V., Reeves, G., et al. (1998) Mortality in vegetarians and non-vegetarians: A collaborative analysis of 8300 deaths among 76,000 men and women in five prospective studies. Public Health Nutrition, 1, 33-41. doi:10.1079/PHN19980006

[18] Cooper, M.J. and Zlotkin, S.H. (1996) Day-to-day variations of transferrin receptor and ferritin in healthy mean and women. The American Journal of Clinical Nutrition, 64, 738-742.

[19] Herbert, V. (1992) Iron disorders can mimic anything, so always test for them. Blood Reviews, 3, 125-132. doi:10.1016/0268-960X(92)90024-K

[20] Shine, J.W. (1997) Microcytic anemia. American Family Physician, 55, 2455-2463.

[21] Ball, M.J. and Bartlett, M.A. (1999) Dietary intake and iron status of Australian vegetarian women. The American Journal of Clinical Nutrition, 70, 353-358.

[22] Due, L., Sinclair, A.J., Mann, N.J., Turner, A. and Ball, M.J. (2000) Selected micronutrient intake and status in men with differing meat intakes, vegetarians and vegans. The Asian Pacific Journal of Clinical Nutrition, 9, 18-23. 
doi:10.1046/j.1440-6047.2000.00129.x

[23] Shaw, N., Chin, C. and Pan, W. (1995) A vegetarian diet rich in soybean products compromises iron status in young students. Journal of Nutrition, 125, 212-219.

[24] Alexander, D., Ball, M.J. and Mann, J. (1994) Nutrient intake and haematological status of vegetarians and agesex matched omnivores. European Journal of Clinical
Nutrition, 4, 538-546.

[25] Harvey, L.J., Armah, C.N., Dainty, J.R., Foxall, R.J., Lewis, D.J. and Langford, N.J. (2005) Impact of menstrual blood loss and diet on iron deficiency among women in the UK. British Journal of Nutrition, 94, 557564. doi:10.1079/BJN20051493 